\title{
Phase-space analysis of fragments formed in heavy-ion collisions
}

\author{
Sakshi Gautam ${ }^{1}$ and Preeti Bansal \\ Department of Physics, Panjab University, Chandigarh-160 014, India.
}

\begin{abstract}
We study the effect of momentum-dependent interactions and a broader Gaussian on multifragmentation. We also look into the details of the fragment structure for a broader Gaussian and momentum-dependent interactions. We find that nucleons forming the fragments belong to same region of the phase space.
\end{abstract}

${ }^{1}$ Email: sakshigautm@gmail.com 


\section{Introduction}

The breaking of colliding nuclei into fragments of different sizes has been studied for quite a long time. The detailed experimental and theoretical studies revealed that the fragmentation is a complex process that depends crucially on the reaction inputs like the bombarding energy as well as impact parameter [1-4]. Various experimental studies offer an unique opportunity to explore the mechanism behind breaking of nuclei into pieces. At the same time, heavy ion reaction can also be used to extract information about the nature of the matter. Some processes like kaon production $[5,6]$ give signal about the softer nature of the matter, whereas others give indication that matter could be stiffer in nature. It is also well accepted that the static equation of state (EOS) cannot describe the heavy-ion reaction adequately. The fate of a reaction depends not only on the density, but also on the momentum space. Therefore, the momentum-dependent interactions play crucial role in the dynamics of a heavy-ion collision. The momentum dependent interactions (MDI) are found to affect the collective flow drastically [7-9]. Due to the reduction in the nucleon-nucleon collisions with MDI, the sub-threshold particle production is also reduced [7] significantly. Some studies are also reported in the literature that focuses on the effect of MDI on multifragmentation [10]. These studies predicted a significant effect of MDI on multifragmentation. These effects were more pronounced at peripheral collisions. Unfortunately, no study has been carried out to look into the details of the fragment structure using momentum dependent interactions. One is interested to understand whether fragments are produced due to coalessence or emerge from the particular region of the phase space. 
In addition, interaction range has also a major role to play in the dynamics of heavy-ion collisions [11-15]. It has a pronounced effect on the collective flow and on its disappearance as well as on multifragmentation $[11,12]$. For example, in Ref. [12] it has been shown that for a broader Gaussian (larger interaction range), the energy of disappearance of flow increases. Similarly, there is a significant effect of interaction range on the fragmentation as well. In Ref. [11] it has been shown that a broader Gaussian leads to reduced fragments. But the details of fragment structure for a broader Gaussian was never studied. We, therefore aim to address

1. The effect of MDI on the fragment structure and

2. The effect of interaction range and to look if fragments then produced belong to certain space or just produced in the reaction without pre-selection.

This study is carried out within the framework of quantum molecular dynamics (QMD) model

\section{The Formalism}

\subsection{Quantum Molecular dynamics (QMD) model}

We describe the time evolution of a heavy-ion reaction within the framework of Quantum Molecular Dynamics (QMD) model [7] which is based on a molecular dynamics picture. This model has been successful in explaining collective flow [16], elliptic flow [17], multifragmentation [18] as well as dense and hot matter [19]. Here each nucleon is represented 
by a coherent state of the form

$$
\phi_{\alpha}\left(x_{1}, t\right)=\left(\frac{2}{L \pi}\right)^{\frac{3}{4}} e^{-\left(x_{1}-x_{\alpha}(t)\right)^{2}} e^{i p_{\alpha}\left(x_{1}-x_{\alpha}\right)} e^{-\frac{i p_{\alpha}^{2} t}{2 m}}
$$

Thus, the wave function has two time dependent parameters $x_{\alpha}$ and $p_{\alpha}$. The total n-body wave function is assumed to be a direct product of coherent states:

$$
\phi=\phi_{\alpha}\left(x_{1}, x_{\alpha}, p_{\alpha}, t\right) \phi_{\beta}\left(x_{2}, x_{\beta}, p_{\beta}, t\right) \ldots
$$

where antisymmetrization is neglected. One should, however, keep in the mind that the Pauli principle, which is very important at low incident energies, has been taken into account. The initial values of the parameters are chosen in a way that the ensemble $\left(A_{T}+A_{P}\right)$ nucleons give a proper density distribution as well as a proper momentum distribution of the projectile and target nuclei. The time evolution of the system is calculated using the generalized variational principle. We start out from the action

$$
S=\int_{t_{1}}^{t_{2}} \mathcal{L}\left[\phi, \phi^{*}\right] d \tau
$$

with the Lagrange functional

$$
\mathcal{L}=\left(\phi\left|i \hbar \frac{d}{d t}-H\right| \phi\right)
$$

where the total time derivative includes the derivatives with respect to the parameters. The time evolution is obtained by the requirement that the action is stationary under the allowed variation of the wave function

$$
\delta S=\delta \int_{t_{1}}^{t_{2}} \mathcal{L}\left[\phi, \phi^{*}\right] d t=0
$$

If the true solution of the Schrödinger equation is contained in the restricted set of wave function $\phi_{\alpha}\left(x_{1}, x_{\alpha}, p_{\alpha}\right)$, this variation of the action gives the exact solution of the 
Schrödinger equation. If the parameter space is too restricted, we obtain that wave function in the restricted parameter space which comes close to the solution of the Schrödinger equation. Performing the variation with the test wave function (2), we obtain for each parameter $\lambda$ an Euler-Lagrange equation;

$$
\frac{d}{d t} \frac{\partial \mathcal{L}}{\partial \dot{\lambda}}-\frac{\partial \mathcal{L}}{\partial \lambda}=0
$$

For each coherent state and a Hamiltonian of the form,

$$
H=\sum_{\alpha}\left[T_{\alpha}+\frac{1}{2} \sum_{\alpha \beta} V_{\alpha \beta}\right] \text {, the Lagrangian and the Euler-Lagrange function can be }
$$
easily calculated $[7]$

$$
\begin{gathered}
\mathcal{L}=\sum_{\alpha} \dot{\mathbf{x}}_{\alpha} \mathbf{p}_{\alpha}-\sum_{\beta}\left\langle V_{\alpha \beta}\right\rangle-\frac{3}{2 L m}, \\
\dot{\mathbf{x}}_{\alpha}=\frac{\mathbf{p}_{\alpha}}{m}+\nabla_{p_{\alpha}} \sum_{\beta}\left\langle V_{\alpha \beta}\right\rangle, \\
\dot{\mathbf{p}}_{\alpha}=-\nabla_{\mathbf{x}_{\alpha}} \sum_{\beta}\left\langle V_{\alpha \beta}\right\rangle .
\end{gathered}
$$

Thus, the variational approach has reduced the n-body Schrödinger equation to a set of 6ndifferent equations for the parameters which can be solved numerically. If one inspects the formalism carefully, one finds that the interaction potential which is actually the Brückner G-matrix can be divided into two parts: (i) a real part and (ii) an imaginary part. The real part of the potential acts like a potential whereas imaginary part is proportional to the cross section.

In the present model, interaction potential comprises of the following terms:

$$
V_{\alpha \beta}=V_{l o c}^{2}+V_{l o c}^{3}+V_{C o u l}+V_{Y u k}+V_{M D I}
$$


$V_{l o c}$ is the Skyrme force whereas $V_{C o u l}, V_{Y u k}$ and $V_{M D I}$ define, respectively, the Coulomb, Yukawa and momentum dependent potentials. The Yukawa term separates the surface which also plays the role in low energy processes like fusion and cluster radioactivity [20]. The expectation value of these potentials is calculated as

$$
\begin{aligned}
V_{l o c}^{2}= & \int f_{\alpha}\left(\mathbf{p}_{\alpha}, \mathbf{r}_{\alpha}, t\right) f_{\beta}\left(\mathbf{p}_{\beta}, \mathbf{r}_{\beta}, t\right) V_{I}^{(2)}\left(\mathbf{r}_{\alpha}, \mathbf{r}_{\beta}\right) \\
& \times d^{3} \mathbf{r}_{\alpha} d^{3} \mathbf{r}_{\beta} d^{3} \mathbf{p}_{\alpha} d^{3} \mathbf{p}_{\beta}, \\
V_{l o c}^{3}= & \int f_{\alpha}\left(\mathbf{p}_{\alpha}, \mathbf{r}_{\alpha}, t\right) f_{\beta}\left(\mathbf{p}_{\beta}, \mathbf{r}_{\beta}, t\right) f_{\gamma}\left(\mathbf{p}_{\gamma}, \mathbf{r}_{\gamma}, t\right) \\
& \times V_{I}^{(3)}\left(\mathbf{r}_{\alpha}, \mathbf{r}_{\beta}, \mathbf{r}_{\gamma}\right) d^{3} \mathbf{r}_{\alpha} d^{3} \mathbf{r}_{\beta} d^{3} \mathbf{r}_{\gamma} \\
& \times d^{3} \mathbf{p}_{\alpha} d^{3} \mathbf{p}_{\beta} d^{3} \mathbf{p}_{\gamma} .
\end{aligned}
$$

where $f_{\alpha}\left(\mathbf{p}_{\alpha}, \mathbf{r}_{\alpha}, t\right)$ is the Wigner density which corresponds to the wave functions (eq. 2). If we deal with the local Skyrme force only, we get

$$
V^{\text {Skyrme }}=\sum_{\alpha=1}^{A_{T}+A_{P}}\left[\frac{A}{2} \sum_{\beta=1}\left(\frac{\tilde{\rho}_{\alpha \beta}}{\rho_{0}}\right)+\frac{B}{C+1} \sum_{\beta \neq \alpha}\left(\frac{\tilde{\rho}_{\alpha \beta}}{\rho_{0}}\right)^{C}\right] .
$$

Here A, B and C are the Skyrme parameters which are defined according to the ground state properties of a nucleus. Different values of $\mathrm{C}$ lead to different equations of state. A larger value of $\mathrm{C}(=380 \mathrm{MeV})$ is often dubbed as stiff equation of state.The finite range Yukawa $\left(V_{Y u k}\right)$ and effective Coulomb potential $\left(V_{C o u l}\right)$ read as:

$$
\begin{gathered}
V_{Y u k}=\sum_{j, i \neq j} t_{3} \frac{\exp \left\{-\left|\mathbf{r}_{\mathbf{i}}-\mathbf{r}_{\mathbf{j}}\right|\right\} / \mu}{\left|\mathbf{r}_{\mathbf{i}}-\mathbf{r}_{\mathbf{j}}\right| / \mu}, \\
V_{\text {Coul }}=\sum_{j, i \neq j} \frac{Z_{\text {eff }}^{2} e^{2}}{\left|\mathbf{r}_{\mathbf{i}}-\mathbf{r}_{\mathbf{j}}\right|}
\end{gathered}
$$


The Yukawa interaction (with $t_{3}=-6.66 \mathrm{MeV}$ and $\mu=1.5 \mathrm{fm}$ ) is essential for the surface effects. The momentum-dependent interactions (MDI) are obtained by parameterizing the momentum dependence of the real part of the optical potential. The final form of the potential reads as $[7]$

$$
U^{M D I} \approx t_{4} \ln ^{2}\left[t_{5}\left(\mathbf{p}_{1}-\mathbf{p}_{2}\right)^{2}+1\right] \delta\left(\mathbf{r}_{1}-\mathbf{r}_{2}\right)
$$

Here $t_{4}=1.57 \mathrm{MeV}$ and $t_{5}=5 \times 10^{-4} \mathrm{MeV}^{-2}$. A parameterized form of the local plus MDI potential is given by

$$
U=\alpha\left(\frac{\rho}{\rho_{o}}\right)+\beta\left(\frac{\rho}{\rho_{o}}\right)+\delta \ln ^{2}\left[\epsilon\left(\rho / \rho_{o}\right)^{2 / 3}+1\right] \rho / \rho_{o}
$$

The parameters $\alpha, \beta, \gamma, \delta$ and $\epsilon$ are listed in Ref. [7]. The momentum-dependent part of the interaction acts strongly in the cases where the system is mildly excited. In this case, the MDI is reported to generate a lot more fragments compared to the static equation of state. The relativistic effect does not play role in low incident energy of present interest $[21]$.

The phase space of the nucleons is stored at several time steps and this is clustered using minimum snapping tree method that binds the nucleons if they are closer than 4 fm.

\section{Results and Discussion}

We simulated the reactions of ${ }^{58} \mathrm{Ni}+{ }^{58} \mathrm{Ni}$ and ${ }^{197} \mathrm{Au}+{ }^{197} \mathrm{Au}$ for $100 \mathrm{MeV} /$ nucleon at central $(\hat{b}=0.2)$ and peripheral $(\hat{b}=0.8)$ colliding geometry. For the present study, we used stiff (Hard), soft (Soft), soft with momentum dependent interactions (SMD) equations of state. 

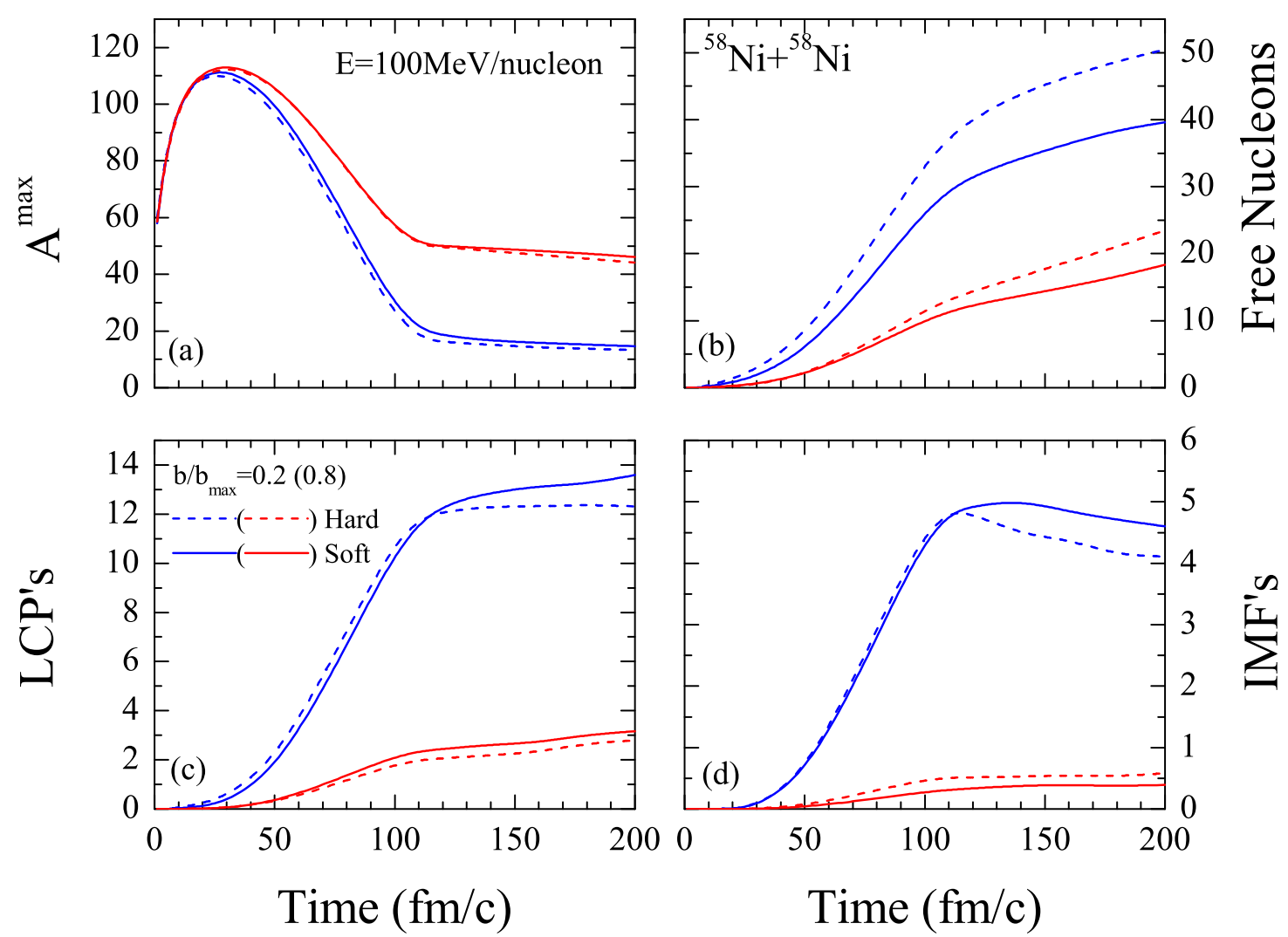

Figure 1: The time evolution of $\mathrm{A}^{\max }$, free nucleons, LCPs and IMFs for the reaction of ${ }^{58} \mathrm{Ni}+{ }^{58} \mathrm{Ni}$ at incident energy of $100 \mathrm{MeV} /$ nucleon at central $(\hat{b}=0.2)$ and peripheral $(\hat{b}$ $=0.8)$ for Soft and Hard EOS. Lines are explained in the text.

The standard energy-dependent Cugnon cross section is used along with two different Gaussian widths, i.e., $\mathrm{L}=1.08 \mathrm{fm}^{2}\left(\mathrm{~L}^{\text {norm }}\right)$ and $2.16 \mathrm{fm}^{2}\left(\mathrm{~L}^{\text {broad }}\right)$.

In Fig. 1, we display the time evolution of $\mathrm{A}^{\max }$, free nucleons, $\operatorname{LCPs}(2 \leq \mathrm{A} \leq 4)$ and IMFs $(5 \leq \mathrm{A} \leq \mathrm{A} / 3)$ for the reaction of ${ }^{58} \mathrm{Ni}+{ }^{58} \mathrm{Ni}$ at $\hat{b}=0.2$ and incident energy of $100 \mathrm{MeV} /$ nucleon. The purpose of showing different mass windows is to identify the 

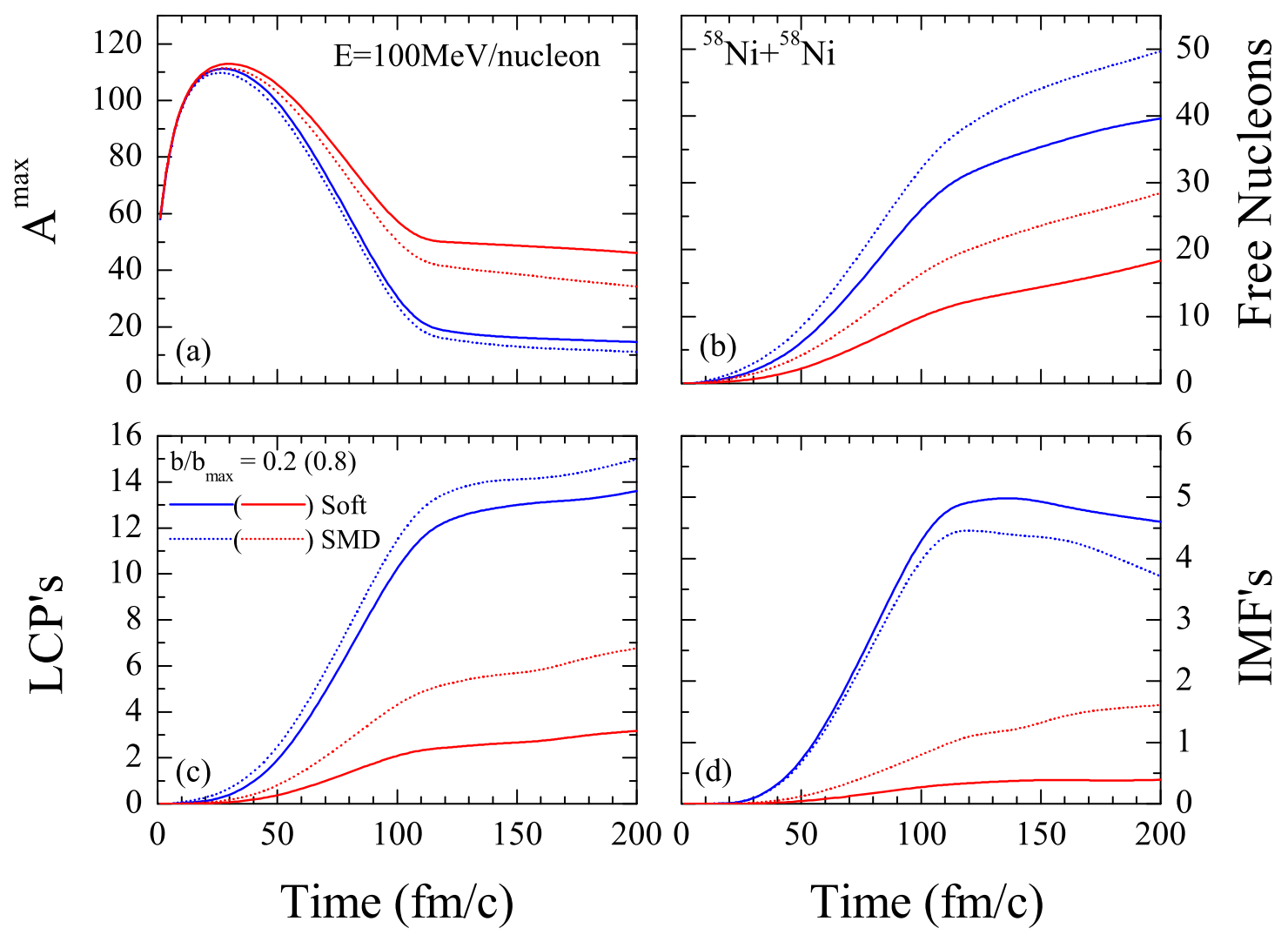

Figure 2: Same as Fig. 1, but for Soft and SMD.

different phenomena that may appear in one window but not in other mass range. The $\mathrm{A}^{\max }$ will give a possibility to look for the fusion (if any), whereas the emission of free nucleons will show the disassembly and hence vaporization of the nuclear matter. For the central collision of $\hat{b}=0.2$ (blue lines) we see from Fig.1(a) that $\mathrm{A}^{\max }$ first increases with time, reaches maximum (about 116 which is $\mathrm{A}^{\text {projectile }}+\mathrm{A}^{\text {target }}$ ) at about 20-40 $\mathrm{fm} / \mathrm{c}$ when the matter is highly compressed and then decreases during the later stages at about $120 \mathrm{fm} / \mathrm{c}$. The effect of EOS is negligible on $\mathrm{A}^{\max }$ (solid and dashed lines) as predicted in Ref. [2]. From Fig. 1(b), (c), and (d), we find that free nucleons, LCPs, and 

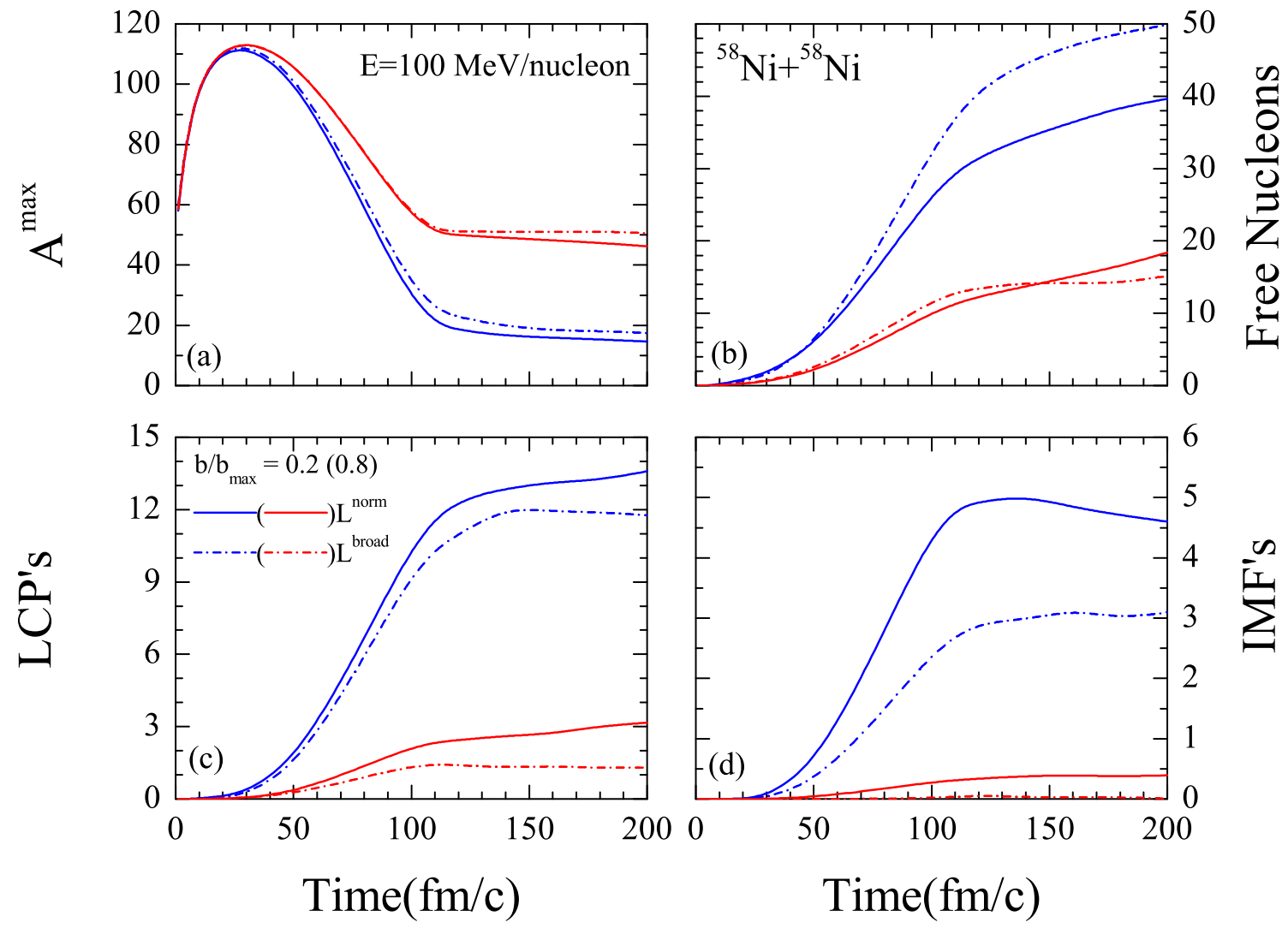

Figure 3: Same as Fig. 1, but for $\mathrm{L}^{\text {norm }}$ and $\mathrm{L}^{\text {broad }}$.

IMFs increases with time. This is because the excited compound nucleus decays by the emission of nucleons and fragments. As a result, free nucleons, LCPs, and IMFs display a constant rise in their multiplicities. The constant emission of free nucleons with time suggests that hot fragments are cooling down. The emission of free nucleons, LCPs, and IMFs starts at around $50 \mathrm{fm} / \mathrm{c}$. We also find a significant effect of EOS on the production of free nucleons, LCPs, and IMFs. We find that number of LCPs/IMFs is larger in the case of soft EOS compared to hard EOS (see blue and red lines). This is because of the fact that soft matter can be easily compressed. As a result, a greater density can be 

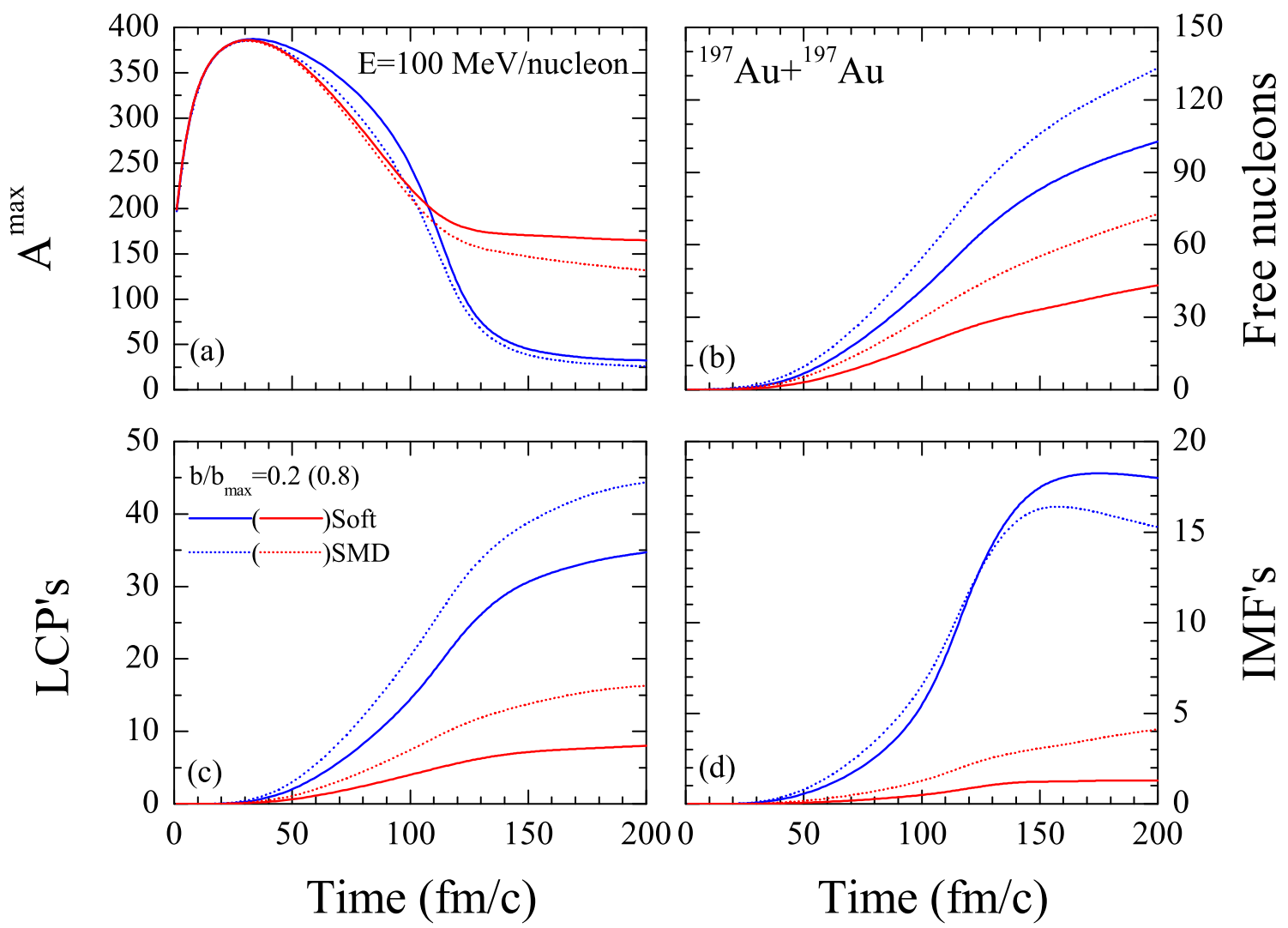

Figure 4: Same as Fig. 2, but for the reaction of ${ }^{197} \mathrm{Au}+{ }^{197} \mathrm{Au}$.

achieved, which in turn leads to the large number of IMFs compared to that in hard case.

For the peripheral collision of $\hat{b}=0.8$ (red lines), we find that $\mathrm{A}^{\max }$, free nucleons, and LCPs show similar behavior as that for central collision except that the number of free nucleons and LCPs are now significantly reduced. This is because of the fact that less density is achieved in peripheral collisions and therefore, the number of IMFs is also greatly reduced in peripheral collisions (both for soft and hard EOS) as the static soft and hard EOS are not able to break the initial correlations among the nucleons and hence no IMFs are emitted. 

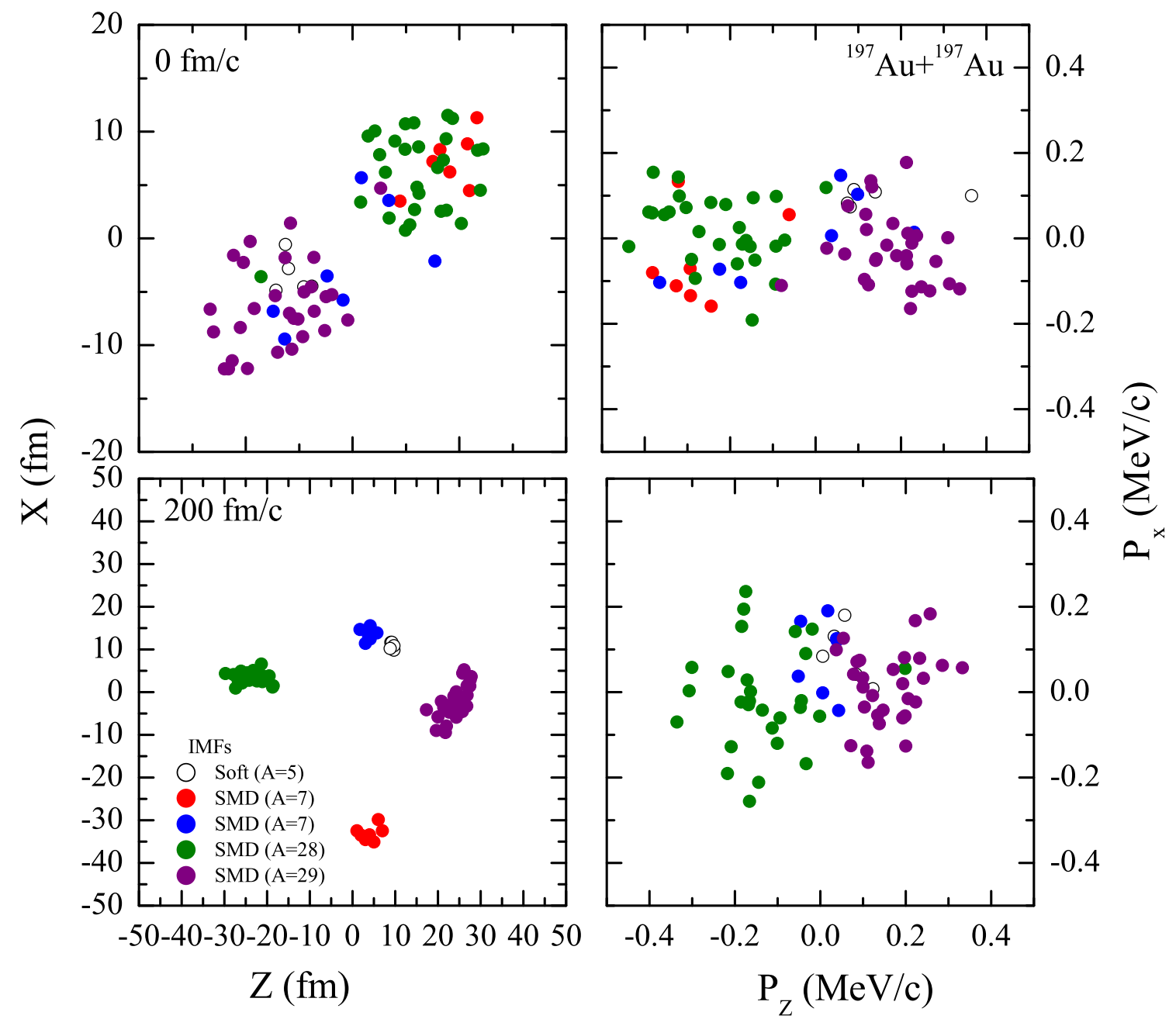

Figure 5: The phase space of the nucleons forming the fragments(IMFs) in the reaction of ${ }^{197} \mathrm{Au}+{ }^{197} \mathrm{Au}$ with Soft and SMD EOS at 0 and $200 \mathrm{fm} / \mathrm{c}$. Symbols are explained in the text.

In Fig. 2, we display the effect of momentum dependent interactions on the production of $\mathrm{A}^{\mathrm{max}}$, free nucleons, LCPs, and IMFs at $\hat{b}=0.2$ and 0.8 . We find that $\mathrm{A}^{\mathrm{max}}$ is nearly same for Soft and SMD (solid and dotted line) at central collisions whereas the difference increases at peripheral collisions. This is because in central collisions, the nucleon-nucleon 
collisions are more frequent which results in complete destruction of the initial correlations. Therefore, an additional repulsion (due to MDI) does not alter the results. We also see that number of free nucleons and LCPs increases with momentum dependent interactions due to additional destruction of the remaining correlations (at both central and peripheral collisions). On the other hand, the role of MDI in peripheral collisions is dominant. This is because in the production of IMFs, the additional MDI breaks the heavy fragments into larger number of intermediate mass fragments leading to a lot of IMFs.

In Fig. 3, we display the effect of interaction range on the production of $\mathrm{A}^{\max }$, free nucleons, LCPs, and IMFs by using two different widths of Gaussian, that is, $\mathrm{L}^{\text {norm }}$ (4.33 $\left.\mathrm{fm}^{2}\right)$ and $\mathrm{L}^{\text {broad }}\left(8.66 \mathrm{fm}^{2}\right)$. We find that the width of Gaussian has a considerable impact on fragmentation. As we change the Gaussian width (L) from $4.33 \mathrm{fm}^{2}$ to $8.66 \mathrm{fm}^{2}$, the multiplicity of IMFs is greatly reduced. Owing to its largest interaction range, an extended wave packet ( $\left.\mathrm{L}^{\text {broad}}\right)$ connects a large number of nucleons in a fragment and as a result it generates heavier fragments compared to what is obtained with a smaller width. It is worth mentioning here that the width of the Gaussian has a considerable effective on the collective flow as well as on the pion production [12-15].

In Fig. 4 we display the effect of MDI on the reactions of ${ }^{197} \mathrm{Au}+{ }^{197} \mathrm{Au}$ at $\hat{b}=0.2$ and 0.8 for $100 \mathrm{MeV} /$ nucleon. A similar behavior of all the quantities is obtained as that for the reaction of ${ }^{58} \mathrm{Ni}+{ }^{58} \mathrm{Ni}$. From Fig. 4, we see that now $\mathrm{A}^{\text {max }}$ reaches a maximum value of (394) which is the total mass of the system at the highly dense phase of the reaction. Moreover the number of free nucleons, LCPs, and IMFs are also increased as that in case of ${ }^{58} \mathrm{Ni}+{ }^{58} \mathrm{Ni}$ reaction due to increase in the system mass. From Fig. 4 we also see that number of IMFs are more in case of SMD as that in case of soft (static) 

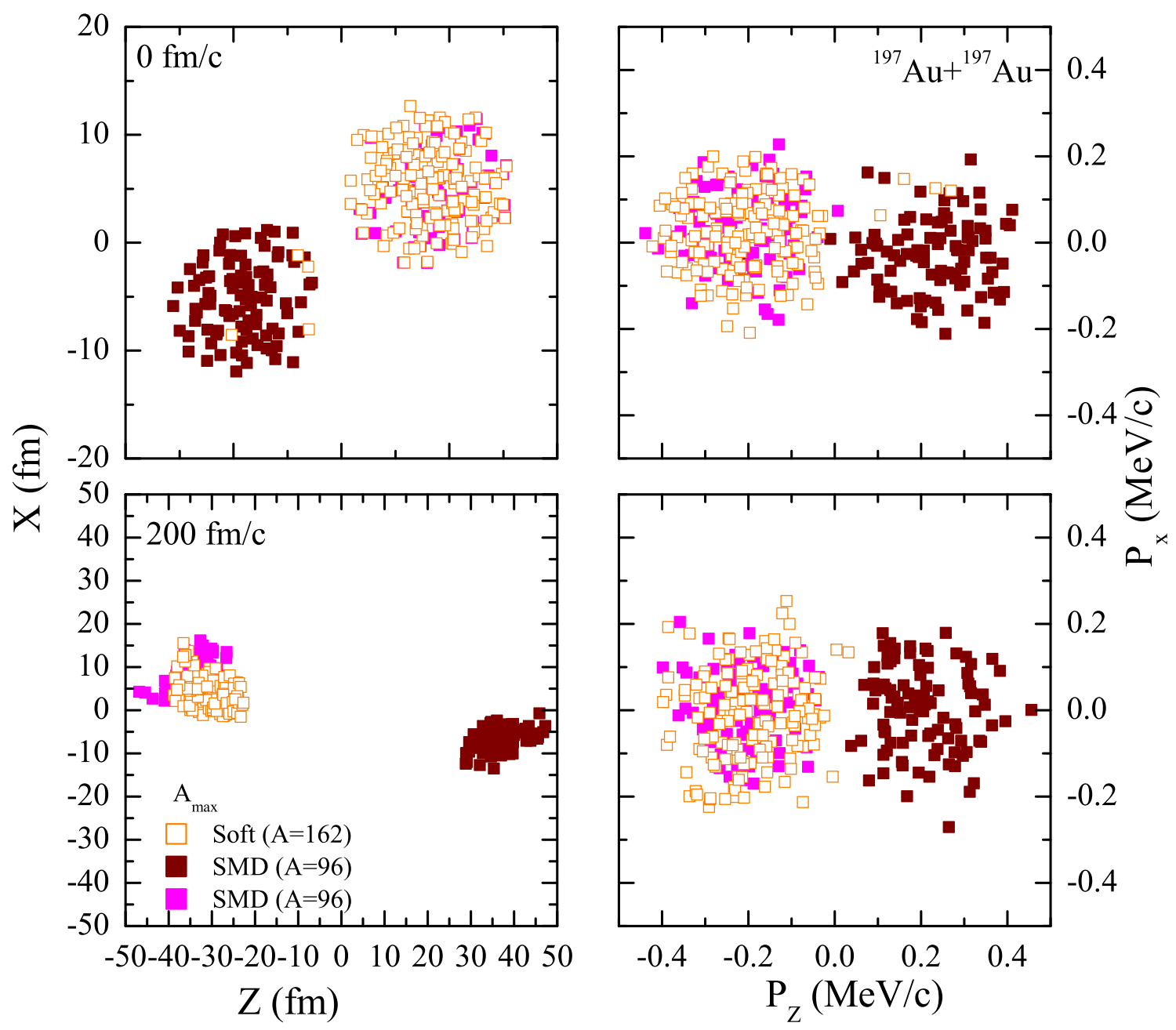

Figure 6: Same as Fig. 5, but for the $\mathrm{A}^{\max }$ formed with Soft and SMD EOS.

EOS because of the destruction of initial correlations due to the repulsive momentum dependent interactions as discussed previously. We further investigate the details of the fragments formed in static and MDI interactions.

In Fig. 5, we display the phase space of those nucleons which form IMFs in case of Soft and SMD EOS at initial time $(0 \mathrm{fm} / \mathrm{c})$ and the end of reaction $(200 \mathrm{fm} / \mathrm{c})$. Left (right) panels display the coordinate (momentum) space. Solid (open) circles represent 

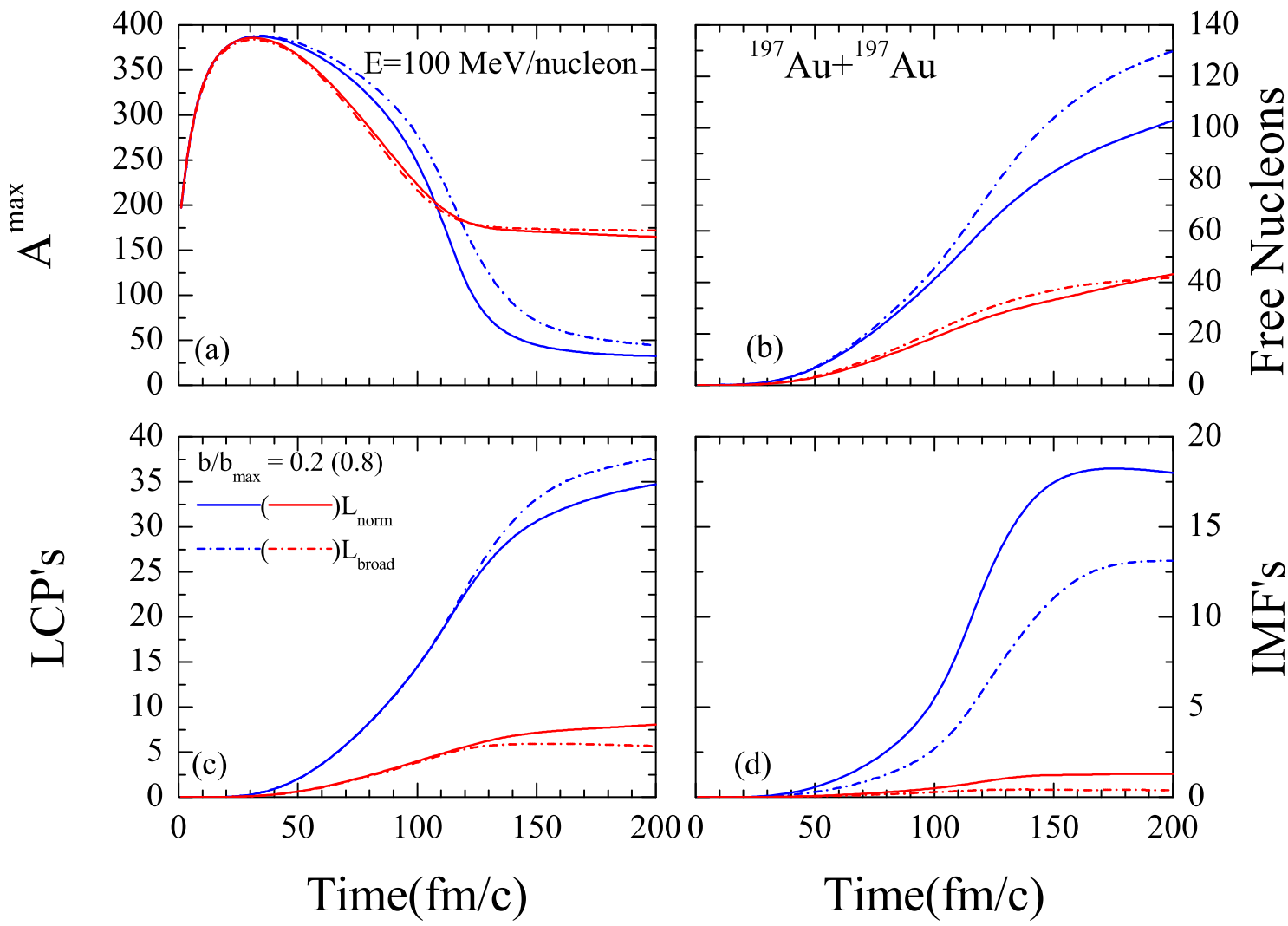

Figure 7: Same as Fig. 3, but for the reaction of ${ }^{197} \mathrm{Au}+{ }^{197} \mathrm{Au}$.

SMD (Soft) EOS. From the Fig., we see that nucleons forming an IMF in case of Soft EOS belong to same region of coordinate space (see open circles). In case of MDI also, most of the nucleons which form the IMFs are coming from the same region (closed circles).

In Fig. 6, we display the phase space of the nucleons forming the $\mathrm{A}^{\mathrm{max}}$ in case Soft and SMD EOS. We see that for the formation of $\mathrm{A}^{\max }$, the participating nucleons belong to the same region of phase space. We also see that $\mathrm{A}^{\max }$ in case of SMD is small as compared to that in case of static one.

In Fig. 7, we display the effect of interaction range on the production of $\mathrm{A}^{\mathrm{max}}$, free 

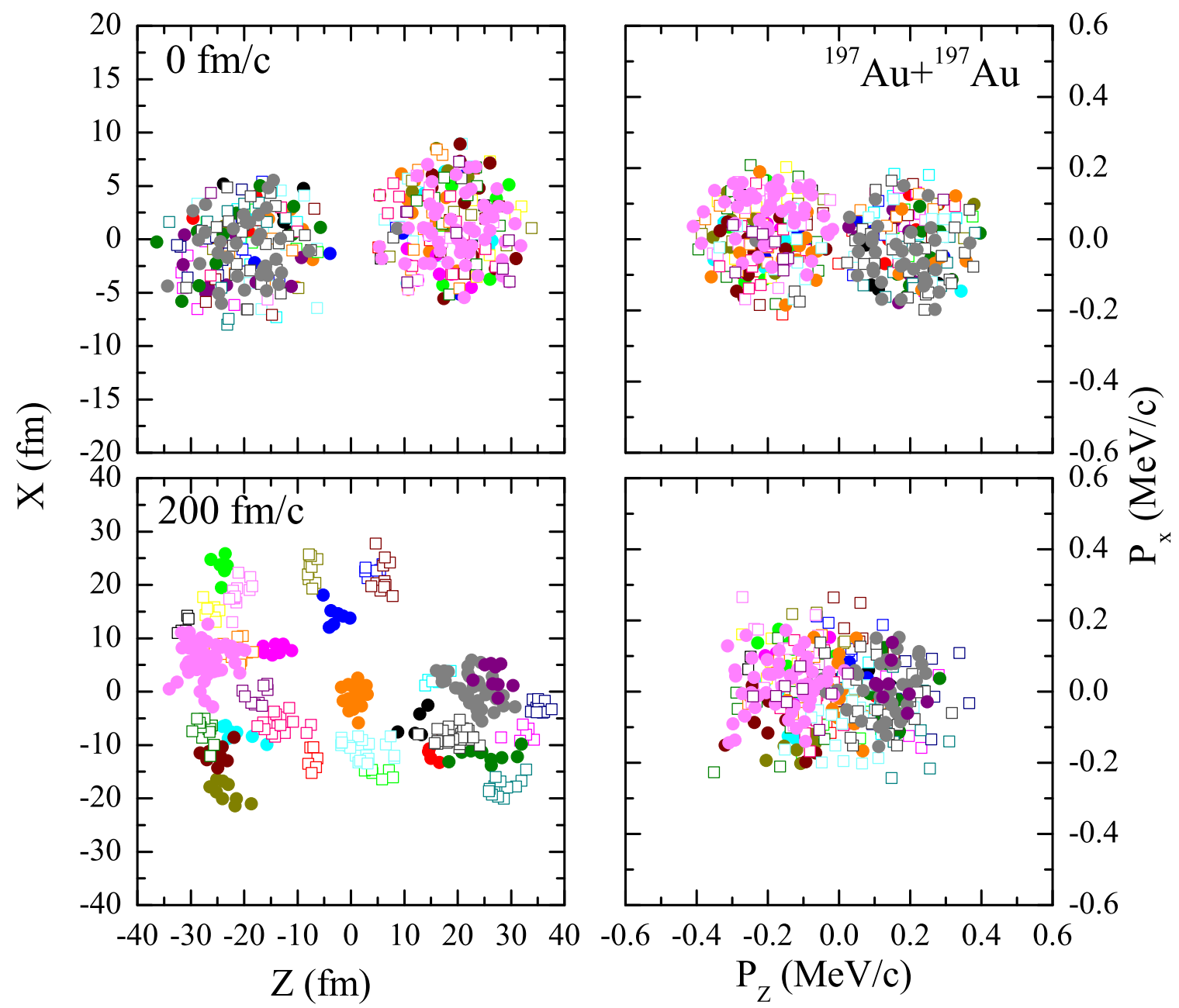

Figure 8: The phase space of the nucleons forming the fragments (IMFs) in the reaction of ${ }^{197} \mathrm{Au}+{ }^{197} \mathrm{Au}$ with $\mathrm{L}^{\text {norm }}$ and $\mathrm{L}^{\text {broad }}$ at 0 and $200 \mathrm{fm} / \mathrm{c}$. Solid (open) symbols are for $\mathrm{L}^{\text {broad }}\left(\mathrm{L}^{\text {norm }}\right)$.

nucleons, LCPs, and IMFs by using two different widths of Gaussian, that is, $\mathrm{L}^{\text {norm }}(4.33$ $\left.\mathrm{fm}^{2}\right)$ and $\mathrm{L}^{\text {broad }}\left(8.66 \mathrm{fm}^{2}\right)$ for the reaction of ${ }^{197} \mathrm{Au}+{ }^{197} \mathrm{Au}$. We find the similar effect of interaction range of the fragment production as for the reaction of ${ }^{58} \mathrm{Ni}+{ }^{58} \mathrm{Ni}$, i.e, with broader Gaussian, the IMF's production is reduced. To have a further insight into the 
fragment structure, that is, whether the nucleons forming a fragment when we increase the interaction range belong to same region of phase space or not, in Fig. 8, we display the phase space of the nucleons which are forming the IMFs both with $L^{\text {norm }}$ and $\mathrm{L}^{\text {broad }}$ at $0 \mathrm{fm} / \mathrm{c}$ and $200 \mathrm{fm} / \mathrm{c}$. We find that the nucleons which are forming the fragment belong to same region of phase space.

\section{Summary}

We studied the effect of momentum-dependent interactions and a broader Gaussian on multifragmentation. We also investigated the details of the fragment structure for a broader Gaussian and momentum-dependent interactions. We find that nucleons forming the fragments belonged to the same region of phase space.

\section{Acknowledgement}

This work is done under the supervision of Dr. Rajeev K. Puri, Department of Physics, Panjab University, Chandigarh, India. This work has been supported by a grant from Centre of Scientific and Industrial Research (CSIR), Govt. of India.

\section{References}

[1] J. Aichelin and H Stöcker, Phys. Lett. B 176, 14 (1986).

[2] S. Kumar et al., Phys. Rev. C 58, 320 (1998); ibid. C 58, 1618 (1998); ibid. C 78, $064602(2008)$ 
[3] R. K. Puri et al., Phys. Rev. C 54, 28 (1996); ibid. C 57, 2744 (1998).

[4] C. Williams et al., Phys. Rev. C 55, R2132 (1997); M. B. Tsang et al., Phys. Rev. Lett. 71, 1502 (1993); Y. K. Vermani et al., Phys. Rev. C 79, 064613 (2009); ibid., Eur. Phys. Lett. 85, 062001 (2009); ibid., J. Phys. G: Nucl. Part. Phys. 37, 015105 (2010); ibid. G 36, 105103 (2009).

[5] C. Hartnack, H. Oeschler and J. Aichelin, Phys. Rev. Lett. 96, 012302 (2006).

[6] C. Fuchs et al., Phys. Rev. Lett. 86, 1974 (2001); S. W. Huang et al., Phys. Lett. B 298, 41 (1998); ibid., Prog. Nucl. Part. Phys. 30, 105 (1993); G. Batko et al., J. Phys. G: Nucl. Part. Phys. 20, 461 (1994).

[7] J. Aichelin, Phys. Rep. 202, 233 (1991).

[8] J. Aichelin, A. Rosenhauer, G. Peilert, H. Stöcker, and W. Greiner, Phys. Rev. Lett. 58, 1926 (1987).

[9] A. D. Sood and R. K. Puri, Eur. Phys. J. A 30, 571 (2006).

[10] G. Peilert, H. Stöcker, W. Greiner, A. Rosenhauer, A. Bohnet, and J. Aichelin, Phys. Rev. C 39, 1402 (1989).

[11] C. Hartnack et al., E. Phys. J. A 1, 151 (1998).

[12] S. Gautam et al., J. Phys. G: Nucl. Part. Phys. 37, 085102 (2010); S. Gautam et al., Phys. Rev. C 82, 014604 (2011); ibid. C 83, 014603 (2011).

[13] C. Hartnack et al., Nucl. Phys. A 580, 643 (1994). 
[14] D. Klakow, G. Welke and W. Bauer, Phys. Rev. C 48, 1982 (1993).

[15] C. Hartnack, J. Jaenicke, L. Sehn, H. Stöcker, and J. Aichelin, Nucl. Phys. A580, $643(1994)$.

[16] A. D. Sood et al., Phys. Lett. B 594, 260 (2004); ibid., Phys. Rev. C 70, 034611 (2004); ibid. C 69, 054612 (2004); ibid. C 73, 067602 (2006); ibid. C 79, 064618 (2009); R. Chugh et al., Phys. Rev. C 82, 014603 (2010); S. Goyal et al., Nucl. Phys. A 853, 164 (2011); V. Kaur et al., Phys. Lett. B 697, 512 (2011).

[17] S. Kumar et al., Phys. Rev. C 81, 014601 (2010); ibid. C 81, 014611 (2010).

[18] J. Dhawan et al., Phys. Rev. C 74, 057901 (2006); ibid. C 74, 057610 (2006).

[19] C. Fuchs et al., J. Phys. G: Nucl. Part. Phys. 22, 131 (1996); Y. K. Vermani et al., Nucl. Phys. A 847, 243 (2010).

[20] R. K. Puri et al., J. Phys. G: Nucl. Part. Phys. 18, 903 (1992); ibid. G 18, 1533 (1992); ibid., Eur. Phys. J. A 3, 277 (1998); ibid. A23, 429 (2005); ibid. A 8, 103 (2008); S. S. Malik et al, Pramana J. Phys. 32, 419 (1989); I. Dutt et al., Phys. Rev. C 81, 064608 (2010); ibid. C 81, 064609 (2010); ibid. C 81, 047601 (2010); ibid. C 81, 044615 (2010).

[21] E. Lehmann et al., Phys. Rev. C 51, 2113 (1995); ibid. Prog. Part. Nucl. Phys. 30, 219 (1993). 